\title{
Exon Skipping during Splicing of Dystrophin mRNA Precursor due to an Intraexon Deletion in the Dystrophin Gene of Duchenne Muscular Dystrophy Kobe
}

\author{
Masafumi Matsuo, Takehiro Masumura, * Hisahide Nishio, Toshihiro Nakajima, Yoshihiko Kitoh, \\ Toru Takumi, Junichi Koga, ${ }^{*}$ and Hajime Nakamura \\ Department of Pediatrics, Kobe University School of Medicine, 7-5-1 Kusunokicho, Chuo, Kobe 650, Japan; and *Biotechnology \\ Research Laboratories, JCR Pharmaceuticals Co., Ltd., 3-2-1 Takatsukadai, Nishi, Kobe 651-22, Japan
}

\begin{abstract}
Recent molecular studies have shown that in a patient with Duchenne muscular dystrophy (DMD) Kobe, the size of exon 19 of the dystrophin gene was reduced to $36 \mathrm{bp}$ due to the deletion of 52 bp out of 88 bp of the exon. The consensus sequences at the $5^{\prime}$ and $3^{\prime}$ splice sites of exon 19 were unaltered (Matsuo, M., et al. 1990. Biochem. Biophys. Res. Commun. 170:963-967). To further elucidate the molecular nature of the defect, we examined the primary structure of cytoplasmic dystrophin mRNA of the DMD Kobe patient across the junctions of exons 18, 19, and 20 by gel electrophoresis and sequencing of polymerase chain reaction-amplified cDNA. The mRNA coding for dystrophin was reverse transcribed using random primers, and the cDNA was then enzymatically amplified in vitro. The targeted fragment was smaller than expected from the genomic DNA analysis. By sequencing of the amplified product, we found that exon 18 was joined directly to exon 20 , so that exon 19 was completely absent, suggesting that this exon was skipped during processing of the dystrophin mRNA precursor. All other bases in the amplified product were unaltered. Therefore, the data strongly suggest that the internal exon deletion generates an abnormally spliced mRNA in which the sequence of exon 18 is joined to the sequence of exon 20 . We propose that the deletion is responsible for abnormal processing of the DMD Kobe allele. This finding has important implications regarding the determinants of a functional splice site. (J. Clin. Invest. 1991. 87:2127-2131.) Key words: Duchenne muscular dystrophy • exon skipping • dystrophin mRNA • frame shift mutation
\end{abstract}

\section{Introduction}

Duchenne muscular dystrophy (DMD) ${ }^{1}$ is a frequent ( 1 per 3,500 live male births), $x$-linked, fatal defect in the dystrophin gene, which is over $2,500 \mathrm{~kb}$ long. Dystrophin is encoded by a 14-kb RNA transcript that is processed from more than 70 exons. As a result, $<1 \%$ of the total gene is transcribed to ma-

Address correspondence to Dr. Masafumi Matsuo, c/o Prof. Uta Francke, Howard Hughes Medical Institute, Beckman Center for Molecular and Genetic Medicine, Stanford University Medical Center, Stanford, CA 94305-5428.

Received for publication 12 November 1990 and in revised form 16 January 1991.

1. Abbreviations used in this paper: DMD, Duchenne muscular dystrophy; $\mathrm{PCR}$, polymerase chain reaction.

J. Clin. Invest.

(c) The American Society for Clinical Investigation, Inc.

$0021-9738 / 91 / 06 / 2127 / 05 \$ 2.00$

Volume 87, June 1991, 2127-2131 ture mRNA and the large amount of splicing that takes place during transcription probably contributes to the high levels of mutation which are observed (reviewed in references 1 and 2).

The splicing of mRNA precursors requires at least two conserved sequences at the ends of introns: the $5^{\prime}$ splice-site consensus sequence 5'-CAG:GTAAGT-3' (where the colon denotes the site of cleavage and ligation) and the $3^{\prime}$ splice-site consensus sequence CAG:G (3). Additionally, as yet uncharacterized sequence elements in mRNA precursors are probably involved in the regulation of splicing. In vitro studies have shown that a relatively long adjacent exon sequence is required for efficient utilization of a splice site $(4,5)$. Most naturally occurring mutations at $5^{\prime}$ consensus sequences inactivate the authentic $5^{\prime}$ splice-site and abolish normal splicing. Certain mutations cause an exon to be skipped during RNA splicing (6-8). However, exon skipping due to a deletion mutation within a nonconsensus exon sequence has never been reported.

Both the large size and the low abundance $(0.01 \%$ of total muscle RNA) of dystrophin message make it very difficult to detect the transcript in diagnostic muscle biopsy samples by Northern hybridization (1). In some patients at least, the message can be detected by in situ hybridization with a probe from the $5^{\prime}$ end of the gene (9). Unlike Northern blot analysis, which requires extensively purified, intact, or high molecular mass RNA, the polymerase chain reaction (PCR) requires only that the average molecular mass of the template mRNA should be slightly greater than the largest fragment amplified. Dystrophin mRNA, which is converted to cDNA by reverse transcriptase, can be detected not only in skeletal muscle but also in nonmuscular tissues by using PCR (10). Furthermore, it is known that many isoforms of dystrophin are present due to alternative splicing of the same precursor mRNA $(11,12)$.

We have identified a 52-bp deletion near the $3^{\prime}$ end of the exon 19 of the dystrophin gene in cells from a patient with DMD Kobe (13). Because we anticipated that this mutation, like many others near to splice junctions (14), might cause abnormal mRNA splicing, the primary structure of dystrophin mRNA was analyzed by PCR amplification of cDNA. In this paper we demonstrate the exon skipping does indeed occur during splicing of dystrophin mRNA precursor in DMD Kobe.

\section{Methods}

Case. The patient (A.N.) is a 16-yr-old Japanese boy. He was diagnosed as having DMD on the basis of high serum creatine kinase levels and electromyogram and muscle biopsy analyses when he was 5 yr old. He became wheelchair bound at the age of 10 and now needs respiratory support from an artificial ventilator. Peripheral blood DNA analysis was performed using PCR and sequencing of the amplified product. A 52-bp deletion near the $3^{\prime}$ end of exon 19 was identified, as shown in Fig. 1 a (13). A stop codon, TGA, was expected to appear at the 791st codon in exon 20 (the numbering in this paper is according to the 


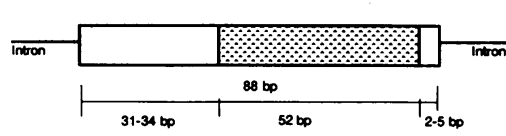

b

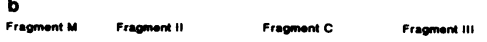

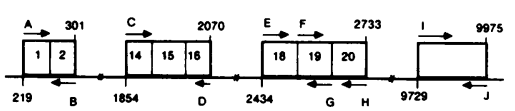

shaded area indicated

the region that has been deleted in DMD Kobe. Four potential sites at which the deletion took place were proposed because of the common nucleotide sequence (GAA) at both ends (13). (b) Position of the different oligonucleotide primers of human dystrophin CDNA. Eight primers named A, B, C, D, E, H, I, and $J$ were used to amplify four fragments of dystrophin cDNA. Fragments M, II, G, and III encompasss exons 1 to 2 , exons 14 to 16 , exons 18 to 20 , and the $3^{\prime}$ terminal region of the coding sequence, respectively. The sizes of fragments M, II, C, and III were 83, 217, 300, and $247 \mathrm{bp}$, respectively. Arrows indicate the positions and the directions of the primers. The sequences of primers A and B and primers C, D, I, and J were reported by Chelly et al. (12) and Muntoni et al. (17), respectively. To amplify exons from 18 to 20, primers $E$ and $H$ were used. Primers $F$ and $G$ were used to determine heterogeneous mRNA. The sequences of primers $\mathrm{E}, \mathrm{H}$, $F$, and $G$ are given in the text. Numbers in boxes indicate exon numbers and the numbers outside the boxes are nucleotide numbers according to the skeletal muscle cDNA sequence (15).

skeletal muscle cDNA sequence [15]). The sequences of the last 154 nucleotides of intron 18 and the first 217 nucleotides of intron 19 were identical to those of wild type DNA, and the consensus sequences at the splice sites were entirely normal. Pedigree analysis established the patient had inherited a DMD Kobe gene from his mother (13). The patient was found to lack dystrophin completely by both immunofluorescence and immunoblotting studies on muscle samples (Arahata, K., personal communication).

Synthesis of $c D N A$. A muscle sample was obtained from the patient's biceps at biopsy, and a control muscle sample from quadriceps was obtained during an orthopedic operation. Total RNA was isolated from muscle samples by guanidinium thiocyanate extraction (16). The RNA was purified by centrifugation on a cesium chloride gradient and was reverse transcribed into single-strand cDNA using random hexanucleotide primers and reverse transcriptase according to the manufacturer's instructions (Amersham International, Amersham, UK). The second DNA strand was synthesized on the cDNA. Double-strand cDNA synthesized using a cDNA synthesis kit purchased from Amersham was extracted with phenol/chloroform and ethanol precipitated.

Amplification of $c D N A$. To amplify the region across the junctions of exons 18, 19, and 20 in cDNA (named fragment C), two PCR primers were synthesized on a DNA synthesizer (Applied Biosystems, Inc., Foster City, CA). The 300 bp long target region extended from the last 67 bp of exon sequence 18 to the first 145 bp of exon 20 . To amplify fragment $C$, a primer based on the sequence of exon 18 (5'-GCAGAGTCCTGAATTTGCAA-3' [E]) and a primer complementary to a sequence of exon 20 (5'-AATTGTTGTAGCTGATTATA-3' [H]), were used. To ascertain the presence of the dystrophin transcript, three fragments (M, II, and III) distributed over the dystrophin transcript were designated for amplification. The sequences of the oligonucleotide primers employed for this purpose (A, B, C, D, I, and J) and the sizes of the amplification products have been described elsewhere (12, 17). The positions of the sequences corresponding to the oligonucleotide primers used are shown in Fig. 1 b. An aliquot $(2 \mu \mathrm{l})$ of doublestrand DNA dissolved in $10 \mu \mathrm{l}$ of water was added to a solution (total 20 $\mu$ ) containing $17 \mathrm{mM}\left(\mathrm{NH}_{4}\right)_{2} \mathrm{SO}_{4}, 67 \mathrm{mM}$ Tris- $\mathrm{HCl}(\mathrm{pH} 8.8), 6.7 \mathrm{mM}$ $\mathrm{MgCl}_{2}, 10 \mathrm{mM} \beta$-mercaptoethanol, $170 \mathrm{mg} / \mathrm{ml}$ BSA, $6.8 \mu \mathrm{M}$ EDTA, all four dNTPs (each at $800 \mu \mathrm{M}$ ), Thermus aquaticus DNA polymerase
I (0.5 U) and two oligodeoxynucleotide primers (each at $1 \mu \mathrm{M})(18) .35$ cycles of PCR amplification were carried out on a DNA Thermal Cycler (Perkin-Elmer Cetus, Emeryville, CA) using the following conditions: initial denaturation at $94^{\circ} \mathrm{C}$ for $7 \mathrm{~min}$, subsequent denaturation at $94^{\circ} \mathrm{C}$ for $0.5 \mathrm{~min}$, annealing at $56^{\circ} \mathrm{C}$ for $0.5 \mathrm{~min}$, and extension at $65^{\circ} \mathrm{C}$ for $4 \mathrm{~min}$. The final extension reaction was carried out at $65^{\circ} \mathrm{C}$ for $7 \mathrm{~min}$. An aliquot of amplified DNA was electrophoresed in a $3 \%$ agarose gel and stained with ethidium bromide along with low molecular size DNA standards (HaeII digested $\phi$ X174 phage DNA) (New England Biolabs, Beverly, MA).

Analysis of heterogeneous $m R N A$. Certain mutations that inactivate a 5 ' splice site activate instead a cryptic splice site, causing heterogeneous mRNA to be produced $(19,20)$. To detect any mRNA that might be produced by the activation of cryptic splice site, we used two oligonucleotide primers that were based on sequence of exon 19 ( $5^{\prime}$ GCCATAGAGCGAGAAAAAGCTGA-3'; primerF) and complementary to exon 19 (5'-TCTGAACTTCTCAGCTTTTTCT-3'; primer G) (Fig. $1 b$ ). The amplification and detection methods were as described above. Trace amounts of normally spliced mRNA would be expected if normal splicing was not completely abolished $(19,20,21)$. To detect this, the region of the agarose gel to which this would have migrated after electrophoresis was cut out. Any DNA it contained was purified by using Gene Clean (Bio 101 Co., La Jolla, CA) and the amplification step repeated.

Sequencing of PCR products. The amplified DNA was extracted with phenol/chloroform, precipitated with ethanol, and dissolved in water. The 5 ' termini of the amplified fragments were phosphorylated with T4 polynucleotide kinase (Pharmacia Fine Chemicals, Piscataway, NJ) and subcloned into SmaI-digested pUC118 (Takara Shuzo Co., Kyoto, Japan). The sequence of inserted DNA from three or four clones were determined by the Sanger dideoxy chain-termination method (22) using a Tth DNA sequencing kit (Toyobo Co., Osaka, Japan), and adenosine $5^{\prime}-\left[\alpha^{32} \mathrm{P}\right]$ triphosphate (Amersham). The products of the sequencing reactions were electrophoresed at $45 \mathrm{~W}$ through $6 \%$ polyacrylamide gels which were then dried and exposed to Fuji RX film overnight at room temperature.

\section{Results}

Since the genetic abnormality in DMD Kobe had been identified as a 52-bp deletion near the $5^{\prime}$ splice site of intron 19 (Fig. 1 $a$ ), we considered it likely that the 5 ' splice site would be inactivated and that a cryptic splice site might be activated (3). The primary structure of cytoplasmic mRNA across the junctions of exons 18, 19, and 20 was therefore determined. Traditional methods of constructing a cDNA library and screening with radioactive probes were not feasible because the steady-state levels of dystrophin mRNA are extremely low (1). Electrophoretic analysis of PCR-amplified CDNA and sequencing of dystrophin cDNA across the junctions of exons 18, 19, and 20 were therefore used to determine the precise structure of mRNA.

PCR amplification products were not obtained in the absence of muscle total RNA in the reverse transcription step. Four fragments (M, II, C, and III) of dystrophin CDNA were amplified when total cytoplasmic RNA from normal muscle was used in the reverse transcription step. The determined sizes of these products were close to those predicted (Fig. 2, lanes 3, 5, 7, and 9). Four fragments were also amplified when total RNA of DMD Kobe was used. Since fragments $M$ and III are located at $5^{\prime}$ and $3^{\prime}$ ends of dystrophin cDNA, respectively, this result indicated that the dystrophin transcript from the DMD patient could be reverse transcribed to cDNA. Three of the four amplified fragments from the patient (M, II, and III) were the same sizes as the control samples (Fig. 2, lanes 2, 4, and 8). 


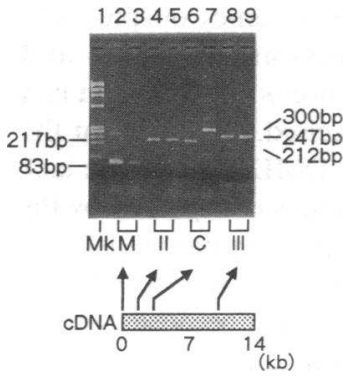

Figure 2. PCR amplification of four regions of dystrophin cDNA. Four fragments (M, II, C, and III) of dystrophin cDNA were amplified. The locations of the amplified fragments distributed on dystrophin cDNA were schematically described. Fragment $M$ is at the $5^{\prime}$ end of cDNA and fragment III is at near 5 ' end of cDNA. In fragments $M$ (lanes 2 and 3) (83 bp), II (lanes 4 and 5) (217 bp), and III (lanes 8 and 9) (247 bp), amplified fragments were the same in the patient and control. Lanes 2, 4, and 8, and lanes 3,5 , and 9 are from patient and control, respectively. The amplified fragment $C$ from the patient (lane 6$)(212 \mathrm{bp}$ ) moved faster than that from the normal individual (lane 7) (300 bp). From its mobility, the length of the patient's amplified fragment was calculated to be $\sim 210$ $\mathrm{bp}$, implying roughly a 90-bp reduction compared to the wild type fragment. Mk refers to HaellI digested $\phi$ X174 phage DNA.

However, fragment $C$ of DMD Kobe ( 210 bp; Fig. 2, lane 6$)$ was $\sim 40$ bp smaller than the length calculated ( $\sim 248 \mathrm{bp})$ from genomic DNA analysis (13) whereas the predicted 300-bp fragment was amplified in the control (Fig. 2, lane 7). The size difference $(\sim 90 \mathrm{bp})$ between the fragment amplified from DMD Kobe $(\sim 210 \mathrm{bp})$ and from the control $(300 \mathrm{bp})$ is approximately the length of exon $19(88 \mathrm{bp})$.

Although a band corresponding to the expected 250-bp fragment was not observed in lane 6 of Fig. 2, the corresponding region was cut out from the gel and any DNA it contained was subjected to PCR amplification using the primers used to amplify fragment $C$. We again failed to detect an amplified 250 -bp product (Fig. $3 a$, lane 1 ). This indicated the complete absence of normal splicing at the $5^{\prime}$ splice site of intron 19 .

Since activation of cryptic splice sites has been reported to result from inactivation of normal $5^{\prime}$ splice sites $(19,20)$, we also looked for abnormally spliced mRNA by attempting specif-

a

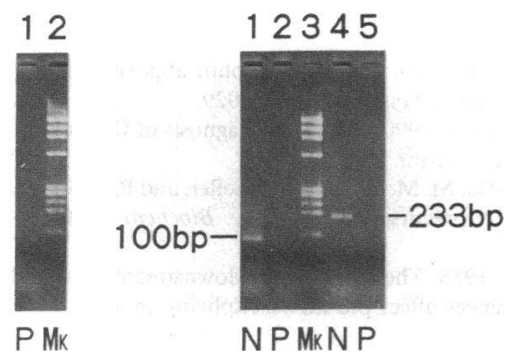

Figure 3. Amplification of heterogeneous cDNA (a) Second amplification of regions across exons 18,19 , and 20. The region of the agarose gel corresponding to $\sim 248$ bp in lane 6 of Fig. 2 was cut out as a 1-mm wide strip. After purification of DNA (see text), DNA was amplified using primers $\mathrm{E}$ and $\mathrm{H}$. No amplification product was observed (lane 1 ). (b) Amplification of segments of cDNA joining exons 18 and 19 or exons 19 and 20 . Segments of cDNA joining exons 18 and 19 or exons 19 and 20 were amplified using primers $E$ and $G$ or $F$ and $H$, respectively. When primers $E$ and $G$ were used, a 100-bp product was observed in the control sample (lane 1 ), but amplified products were not detected in the patient's sample (lane 2). Likewise, a 233-bp product was amplified in the control sample when primers $F$ and $H$ were used (lane 4 ) whereas amplified products were not obtained when cDNA from the patient was used (lane 5). Mk, P, and N refer to marker (HaelII-digested $\phi$ X174 phage DNA), patient and normal, respectively. ically to amplify fragments joining exons 18 and 19 or exons 19 and 20 using combinations of primers $\mathrm{E}$ and $\mathrm{G}$ or $\mathrm{F}$ and $\mathrm{H}$. Once again we failed to detect any amplified DNA (Fig. $3 b$, lanes 2 and 5), suggesting that aberrantly spliced mRNA was not present in patient's muscle cDNA.

The two amplified $C$ fragments were sequenced to see whether the smaller size of the fragment amplified from DMD Kobe mRNA was indeed due to omission of exon 19. Amplified DMD Kobe cDNA was found to be completely devoid of exon 19 , and to have exon 18 precisely joined to exon 20 (Fig. 4). Thus, all 36 bp corresponding to the remnants of exon 19 in the genomic DNA sequence were absent from the cDNA.

The translational reading frame of the resulting mRNA is thus shifted by two bases by the exon skipping, and a stop codon, TGA, appears at the 779th codon in exon 20. This confirms the previously proposed frame-shift explanation for the defect in DMD patients (23). Analysis of the last 67 nucleotides of exon 18 and the first 145 nucleotides of exon 20 did not reveal any additional base changes. Since the internal exon deletion was the only change detected in the sequence of the mutant allele, it is extremely likely that the observed mutation is indeed responsible for skipping of exon 19 during posttranscriptional maturation of the primary transcript.

\section{Discussion}

It is well known that abnormal splicing of mRNA precursors can be induced by genomic DNA mutations in some inherited

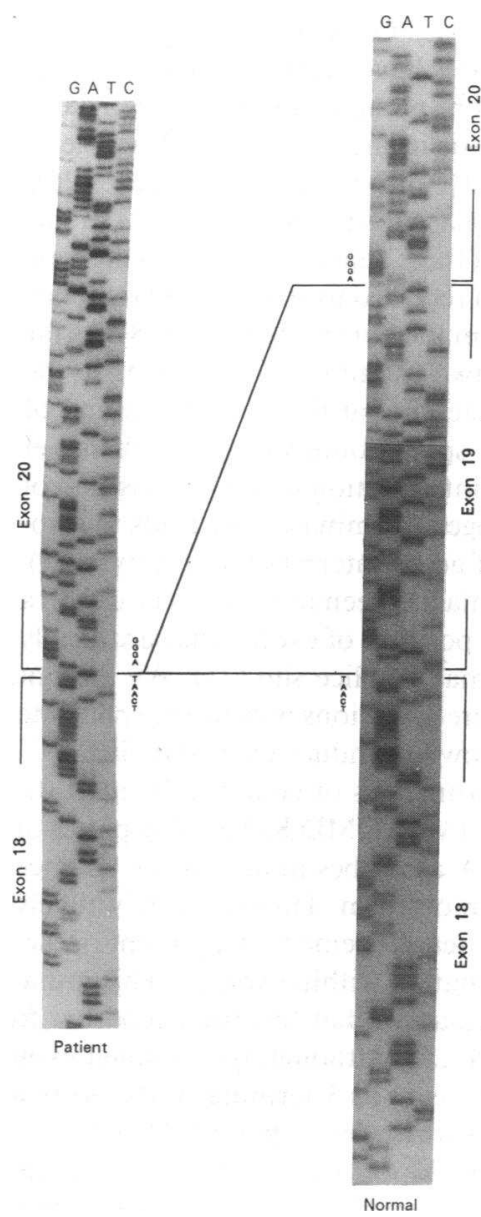

Figure 4. Nucleotide sequences at the junctions of exons 18,19 , and 20 of dystrophin cDNA from the patient and a normal control. Part of nucleotide sequence is shown beside the sequence ladders. The normal sequence (right) is the junction between exon 18 and 20. This nucleotide sequence is identical to that reported by Koenig et al. (15). In the sequence obtained with the sample from the patient (left), the entire 88 bases ( $5^{\prime}$-GCCA---AATG-3') of exon 19 sequence are absent even though 36 bp of exon 19 are present in the genomic DNA and should thus be present in the cDNA. The upstream sequence ( 5 TCAAT- $3^{\prime}$ ) of exon 18 , is directly connected outframe to the downstream sequence (5'-AGGG-3') of exon 20. 
diseases (14). We have previously reported that the dystrophin gene from a DMD Kobe patient has an intraexon deletion near $5^{\prime}$ splice donor site (Fig. 1) (13). Gel electrophoretic analysis of PCR amplified CDNA and cDNA sequencing have now been used to examine the primary structure of dystrophin mRNA. Dystrophin transcripts could be readily detected in diagnostic muscle biopsy samples by amplifying cDNA that had been reverse transcribed using random hexanucleotide primers. We were able to detect four fragments distributed over the dystrophin cDNA, including its $5^{\prime}$ and $3^{\prime}$ ends (Fig. 2). One of the four amplified products from DMD Kobe (fragment C) migrated abnormally upon agarose gel electrophoresis. The size of this fragment $C$, which should encompass the region of exons 18 , 19, and 20, was smaller than expected (Fig. 2, lane 6). DNA sequencing of the amplified product showed that the truncated exon 19 sequence ( $36 \mathrm{bp}$ ) was absent from the dystrophin mRNA (Fig. 4). Thus, the splice site has been inactivated by the mutation without apparently activating a new splice site (Fig. 3). This is the first time that a natural mutation within a nonconsensus exon sequence has been shown to cause exon skipping.

What causes the exon 19 skipping in DMD Kobe? Exon skipping resulting from genomic DNA mutations has been reported in some inherited diseases (14). Mutations affecting either of the conserved sequences at the $5^{\prime}$ donor and $3^{\prime}$ acceptor sites, respectively, induce exon skipping. Most reported cases of exon skipping are due to a single base substitution that changes the universal AG dinucleotide at the $3^{\prime}$ splice site of the intron (14). Point mutations in the consensus sequence at the $5^{\prime}$ splice donor site that lead to genetic disease are very rare (6). Four mutations in the consensus-GTAAGT-heptanucleotide at the $5^{\prime}$ end of intron sequences have been reported to induce skipping of the preceding exon, namely one in the phenylalanine hydroxylase gene (24), one in the pro- $\alpha 2$ (I) collagen gene (25), one in the adenine phosphoribosyltransferase gene (26), and one in the type III procollagen gene (8). An A to G mutation at terminal nucleotide of the 5 ' splice donor site in the type III procollagen gene has been reported to activate cryptic splice sites located downstream from the normal splice site (8). As far as we know, there are only two examples of mutations in the consensus $5^{\prime}-\mathrm{CAG}-3^{\prime}$ trinucleotide at the $3^{\prime}$ terminal end of exons which inactivate the $5^{\prime}$ splice donor site. Grandchamp et al. reported that $\mathrm{a} G$ to $A$ point mutation in the last position of exon 12 of the porphobilinogen deaminase gene leads to skipping of exon 12 in a case of acute intermittent porphyria (7), and one case of $\beta^{+}$-thalassemia has been reported to be due to a G-C transversion at the last position of exon 1 that drastically reduces splicing at the normal $5^{\prime}$ splice site (21). We are not aware of any other cases where mutations outside the splice site consensus sequences are known to induce exon skipping.

Southern hybridization analysis of genomic HindIII-digested DNA from the patient with DMD Kobe using pieces of full-length dystrophin cDNA as probes indicated the absence of restriction fragment polymorphism. Thus it is very unlikely that there is any other major rearrangement of a genomic structure besides the deletion mutation within exon 19. This mutation is unlike any other mutation that has been reported to induce exon skipping $(8,24-26)$. Although the mutated exon 19 has the same five nucleotides at its $3^{\prime}$ terminal as the normal exon 19, the rest of its sequence is quite different. Therefore, it is highly plausible that exon skipping was induced by the intraexon deletion. Reed and Maniatis (27) have reported that different exon sequences can affect the efficiency of splice site selection in an in vitro cis competition assay. Nelson and Green (5) also reported that the sequence within an exon can affect the efficiency with which it is excised. The fact that the all-mRNA products from the mutant DMD Kobe allele lack exon 19 suggests that the truncated exon is disregarded by the splicing machinery. We think it likely that the partial deletion of exon 19 prevents the recognition of the 5 ' splice site of intron 19 because the truncated exon is too small or because it affects a cis-acting factor. This needs to be clarified by further studies, but it has important implications regarding the determinants of functional splice site.

More than $60 \%$ of patients with DMD/BMD (Becker muscular dystrophy) have a partial deletion of the dystrophin gene. Evidence to support the idea that the mRNA reading frame is shifted in cases of DMD due to partial gene deletion (23) has been obtained by Southern blot analysis of genomic DNA. However, some deletion mutations do not appear to alter the reading frame $(28,29)$. The results of genomic DNA analysis of DMD Kobe showed that a 52-bp deletion within exon 19 should cause a frame shift that generates a translational termination signal at the 791st codon in exon 20 (13). In this study on CDNA of DMD Kobe we showed that exon skipping occurred and that the termination signal is in fact located at the 779th codon in exon 20. This difference may explain the fact that the predicted reading frame shifts are not always observed in cases of DMD, since exon skipping could restore the correct reading frame to the (truncated) mature mRNA. Henceforth, mRNA analysis should be used to determine reading frame of dystrophin gene.

\section{Acknowledgments}

We thank Dr. A. Pugsley for advice and for critically reading the manuscript.

This work was supported by grants from the Ministry of Education, Science, and Culture of Japan, the National Center of Neurology and Psychiatry of the Ministry of Health and Welfare of Japan, the Morinaga Hoshikai Foundation, and the Mother and Child's Health Foundation.

\section{References}

1. Hoffman, E. P., and L. M. Kunkel. 1989. Dystrophin abnormalities in Duchenne/Becker muscular dystrophy. Neuron. 2:1019-1029.

2. Beggs, A. H., and L. M. Kunkel. 1990. Improved diagnosis of Duchenne/ Becker muscular dystrophy. J. Clin. Invest. 85:613-619.

3. Padgett, R. A., P. J. Grabowski, M. M. Konarska, S. Seiler, and P. A. Sharp. 1986. Splicing of messenger RNA precursors. Annu. Rev. Biochem. 55:11191150.

4. Furdon, P. J., and R. Kole. 1988. The length of the downstream exon and the substitution of specific sequences affect pre-mRNA splicing in vitro. Mol. Cell. Biol. 8:860-866.

5. Nelson, K. K., and M. R. Green. 1988. Splice site selection and ribonucleoprotein complex assembly during in vitro pre-mRNA splicing. Genes \& Dev. 2:319-329.

6. Jacob, M., and H. Gallinaro. 1989. The 5' splice site: phylogenetic evolution and variable geometry of association with U1RNA. Nucleic Acids Res. 17:21592180.

7. Grandchamp, B., C. Picat, C. Beaumont, P. Wilson, J. C. Deybach, and Y. Nordmann. 1989. A point mutation G-A in exon 12 of the porphobilinogen deaminase gene results in exon skipping and is responsible for acute intermittent porphyria. Nucleic Acids Res. 17:6637-6649.

8. Kontusaari, S., G. Tromp, H. Kuivaniemi, R. L. Ladda, and D. J. Prockop. 1990. Inheritance of an RNA splicing mutation $\left(G^{+11 v 520}\right)$ in the type III procollagen gene (COL3AI) in a family having aortic aneurysms and easy bruisability: phenotypic overlap between familial arterial aneurysms and Ehlers-Danlos syndrome Type IV. Am. J. Hum. Genet. 47:112-120. 
9. Oronzi Scott, M., J. E. Sylvester, T. Heinman-Patterson, Y.-J. Shi, W Fieles, H. Stedman, A. Burghes, P. Ray, R. Worton, and K. H. Fischbeck. 1988 Duchenne muscular dystrophy gene expression in normal and diseased human muscle. Science (Wash. DC). 239:1418-1420.

10. Chelly, J., J.-C. Kaplan, P. Maire, S. Gautron, and A. Kahn. 1988. Transcription of the dystrophin gene in human muscle and non-muscle tissues. Nature (Lond.). 333:858-860.

11. Feener, C. H., M. Koenig, and L. M. Kunkel. 1989. Alternative splicing of human dystrophin mRNA generates isoforms at the carboxy terminus. Nature (Lond.). 338:509-511.

12. Chelly, J., G. Hamard, A. Koulakoff, J. C. Kaplan, A. Kahn, and Y Berwald-Netter. 1990. Dystrophin gene transcribed from different promoters in neuronal and glial cells. Nature (Lond.). 344:64-65.

13. Matsuo, M., T. Masumura, T. Nakajima, Y. Kitoh, T. Takumi, H. Nishio, J. Koga, and H. Nakamura. 1990. A very small frame-shifting deletion within exon 19 of the Duchenne muscular dystrophy gene. Biochem. Biophys. Res. Commun. 170:963-967.

14. Green, M. R. 1986. Pre-mRNA splicing. Annu. Rev. Genet. 20:671-708

15. Koenig, M., A. P. Monaco, and L. M. Kunkel. 1988. The complete sequence of dystrophin predicts a rod-shaped cytoskeletal protein. Cell. 53:219228.

16. Chirowin, J. M., A. E. Przybyla, R. J. MacDonald, and W. J. Rutter. 1979. Isolation of biologically active ribonucleic acid from sources enriched in ribonuclease. Biochemistry. 18:5294-5299.

17. Muntoni, F., and P. N. Strong. 1989. Transcription of the dystrophin gene in Duchenne muscular dystrophy muscle. FEBS (Fed. Eur. Biochem. Soc.) Lett. 252:95-98.

18. Chamberlain, J. S., R. A. Gibbs, J. E. Ranier, and C. T. Caskey. 1990 Multiplex PCR for the diagnosis of Duchenne muscular dystrophy. In PCR Protocols. A Guide to Methods and Applications. M. A. Innis, D. H. Gelfand, J. J. Sninsky, and T. J. White, editors. Academic Press, Inc., San Diego. 272-281.

19. Treisman, R., S. H. Orkin, and T. Maniatis. 1983. Specific transcription and RNA splicing defects in five cloned $\beta$-thalassaemia genes. Nature (Lond.). 302:591-596.

20. Nakajima, H., N. Kono, T. Yamasaki, K. Hotta, M. Kawachi, M. Kuwa- jima, T. Noguchi, T. Tanaka, and S. Tarui. 1990. Genetic defect in muscle phosphofructokinase deficiency. Abnormal splicing of the muscle phosphofructokinase gene due to a point mutation at the 5'-splice site. J. Biol. Chem. 265:93929395.

21. Vidaud, M., R. Gattoni, J. Stevenin, D. Vidaud, S. Amselem, J. Chibani, J. Rosa, and M. Goossens. 1989. A 5' splice-region G-C mutation in exon 1 of the human $\beta$-globin gene inhibits pre-mRNA splicing: a mechanism for $\beta^{+}$-thalassemia. Proc. Natl. Acad. Sci. USA. 86:1041-1045.

22. Sanger, F., S. Nicklen, and A. R. Coulson. 1977. DNA sequencing with chain terminating inhibitors. Proc. Natl. Acad. Sci. USA. 74:5463-5467.

23. Monaco, A. P., C. J. Bertelson, S. Liechti-Gallati, H. Moser, and L. M. Kunkel. 1988. An explanation for the phenotypic differences between patients bearing partial deletions of the DMD locus. Genomics. 2:90-95.

24. Marvit, J., A. G. DiLella, K. Brayton, F. D, Ledley, K. J. H. Robson, and S. L. C. Woo. 1987. GT to AT transition at a splice site causes skipping of the preceding exon in phenylketonuria. Nucleic Acids Res. 15:5613-5628.

25. Weil, D., M. Bernard, N. Combates, M. K. Wirtz, D. W. Hollister, B Steinmann, and F. Ramirez. 1988. Identification of a mutation that causes exon skipping during collagen pre-mRNA splicing in an Ehlers-Danlos syndrome variant. J. Biol. Chem. 263:8561-8564.

26. Hidaka, Y., T. D. Palella, T. E. O'Toole, S. A. Tarlé, and W. N. Kelley. 1987. Human adenosine phosphoribosyltransferase: identification of allelic mutations at the nucleotide level as a cause of complete deficiency of the enzyme. $J$. Clin. Invest. 80:1409-1415.

27. Reed, R., and T. Maniatis. 1986. A role for exon sequences and splice-site proximity in splice-site selection. Cell. 46:681-690.

28. Gillard, E. F., J. S. Chamberlain, E. G. Murphy, C. L. Duff, B. Smith, A. H. M. Burghes, M. W. Thompson, J. Sutherland, I. Oss, S. E. Bodrug, et al. 1989. Molecular and phenotypic analysis of patients with deletions within the deletion-rich region of the Duchenne muscular dystrophy (DMD) gene. Am. J. Hum. Genet. 45:507-520.

29. Koenig M. A. H Beggs, M. Moyer, S. Scherpf, K Heindrich, T. Bettecken, G. Meng, C. R. Muller, M. Lindrof, H. Kaariainen, et al. 1989. The molecular basis for Duchenne versus Becker muscular dystrophy: correlation of severity with type of deletion. Am. J. Hum. Genet. 45:495-506. 